\title{
Constructing regular self-complementary uniform hypergraphs
}

\author{
Shonda Gosselin \\ Department of Mathematics and Statistics, University of Winnipeg \\ 515 Portage Avenue, Winnipeg, MB R3B 2E9, CANADA \\ E-mail: s.gosselin@uwinnipeg.ca \\ Phone: $011+1-204-786-9346$ \\ Fax: $011+1-204-774-4134$
}

17th February 2011

\begin{abstract}
In this paper, we examine the possible orders of $t$-subset-regular selfcomplementary $k$-uniform hypergraphs, which form examples of large sets of two isomorphic $t$-designs. We reformulate Khosrovshahi and TayfehRezaie's necessary conditions on the order of these structures in terms of the binary representation of the rank $k$, and these conditions simplify to a more transparent relation between the order $n$ and rank $k$ in the case where $k$ is a sum of consecutive powers of 2 . Moreover, we present new constructions for 1-subset-regular self-complementary uniform hypergraphs, and prove that these necessary conditions are sufficient for all $k$, in the case where $t=1$.
\end{abstract}

Key words: Self-complementary hypergraph, Regular hypergraph, Complementing permutation, Large set of $t$-designs

AMS Subject Classification Codes: 05C65, 05B05 05E20, 05 C85.

\section{Introduction}

\subsection{Definitions}

For a finite set $\mathcal{V}$ and a positive integer $k$, let $\mathcal{V}^{(k)}$ denote the set of all $k$-subsets of $\mathcal{V}$. A hypergraph with vertex set $\mathcal{V}$ and edge set $\mathcal{E}$ is a pair $(\mathcal{V}, \mathcal{E})$, in which $\mathcal{V}$ is a finite set and $\mathcal{E}$ is a collection of subsets of $\mathcal{V}$. For a hypergraph $X$ in which every edge has cardinality in a given set of positive integers $K$, the complement $X^{C}$ of $X=(\mathcal{V}, \mathcal{E})$ is the hypergraph $\left(X, \mathcal{E}^{C}\right)$, where $\mathcal{E}^{C}=\bigcup_{k \in K}\left(\mathcal{V}^{(k)} \backslash \mathcal{E}\right)$. An isomorphism between two hypergraphs $X=(\mathcal{V}, \mathcal{E})$ and $X^{\prime}=\left(\mathcal{V}^{\prime}, \mathcal{E}^{\prime}\right)$ is a bijection $\phi: \mathcal{V} \rightarrow \mathcal{V}^{\prime}$ which induces a bijection from $\mathcal{E}$ to $\mathcal{E}^{\prime}$. If such an isomorphism exists, the hypergraphs $X$ and $X^{\prime}$ are said to be isomorphic. A 
hypergraph is self-complementary if it is isomorphic to its complement, and an isomorphism between a self-complementary hypergraph and its complement is called an antimorphism. The set of all antimorphisms of $X$ will be denoted by $\operatorname{Ant}(X)$. An automorphism of a hypergraph $X$ is an isomorphism from $X$ to $X$, and the set of all automorphisms of a hypergraph $X$ form a group, denoted by $\operatorname{Aut}(X)$. The rank of an edge $e$ in a hypergraph is its cardinality $|e|$, and the order of a hypergraph $(\mathcal{V}, \mathcal{E})$ is $|\mathcal{V}|$. A hypergraph $(\mathcal{V}, \mathcal{E})$ is called $k$-uniform (or a $k$-hypergraph) if $\mathcal{E}$ is a subset of $\mathcal{V}^{(k)}$, and the rank of a $k$-uniform hypergraph is $k$. Note that a 2 -hypergraph is a graph. We will often denote the vertex set and edge set of a hypergraph $X$ by $\mathcal{V}(X)$ and $\mathcal{E}(X)$, respectively.

Let $X=(\mathcal{V}, \mathcal{E})$ be a hypergraph with edge ranks in a set of positive integer $K$, let $t \in K$, and let $f \in \mathcal{V}^{(t)}$. We define the t-valency $\operatorname{val}_{X}^{t}(f)$ of $f$ in $X$ to be the number of edges $e \in \mathcal{E}$ containing $f$. A $k$-uniform hypergraph $X$ is called $t$-subset-regular if the $t$-valency of $f$ in $X$ is independent of the choice of $f \in \mathcal{V}^{(t)}$, and hence is called the t-valency of $X$ without ambiguity.

\subsection{History and layout of the paper}

In this paper, we examine the possible orders of $t$-subset-regular self-complementary uniform hypergraphs. The following result gives necessary conditions on the order of these structures. This is actually a corollary to a more general result due to Khosrovshahi and Tayfeh-Rezaie [9], which gave necessary conditions on the order of large sets of $t$-designs. For positive integers $m$ and $n$, let $n_{[m]}$ denote the unique integer in $\{0,1, \ldots, m-1\}$ such that $n \equiv n_{[m]}(\bmod m)$.

Theorem 1.1. [9] Let $t, k$ and $n$ be positive integers such that $t<k \leq n$. If there exists a t-subset-regular self-complementary $k$-hypergraph of order $n$, then there exists an integer a such that $\max \left\{i: 2^{i} \mid k\right\}<a \leq \min \left\{i: 2^{i}>k\right\}$ and

$$
n_{\left[2^{a}\right]} \in\left\{t, t+1, \ldots, k_{\left[2^{a}\right]}-1\right\} .
$$

In Section 2, Theorem 2.1, we refine the result of Theorem 1.1 slightly to show that, for the given integer $a, a-1$ must lie in the support of the binary representation of $k$. Potočnik and Šajna first noted this refinement in the case where the rank $k$ has the form $k=2^{\ell}$ or $k=2^{\ell}+1$ [12]. Theorem 2.1 yields the more transparent necessary conditions in Corollary 2.2 on the order of a $t$-subset-regular self-complementary $k$-hypergraph in the case where $k$ is a sum of consecutive powers of 2 . In particular, we obtain Corollary 2.3, which shows that when $k=2^{\ell+1}-1$ and there exists a $t$-subset-regular self-complementary $k$-hypergraph, then $t \leq n_{\left[2^{\ell+1}\right]}<k$.

The necessary conditions of Theorem 1.1 have been shown to be sufficient in the cases where $k \in\{2,3\}$. The case where $k=2$ was handled constructively by Rao [13], but there is also a proof due to Wilson [15]. Potočnik and Šajna handled the case where $k=3$ and $t=1$ [11], and Knor and Potočnik handled the case where $k=3$ and $t=2$ [10]. In Section 3 , we show that the necessary 
conditions of Theorem 1.1 are sufficient for all $k$, in the case where $t=1$. We obtain the following main result.

Theorem 1.2. Let $k$ and $n$ be positive integers such that $1<k \leq n$. There exists a 1-subset-regular self-complementary $k$-hypergraph of order $n$ if and only if there exists an integer a such that $\max \left\{i: 2^{i} \mid k\right\}<a \leq \min \left\{i: 2^{i}>k\right\}$ and

$$
n_{\left[2^{a}\right]} \in\left\{1,2, \ldots, k_{\left[2^{a}\right]}-1\right\} .
$$

\subsection{Connection to design theory}

There is a connection between $t$-subset-regular hypergraphs and designs. Hence results from design theory are applicable to these hypergraphs and vice versa. A $t-(n, k, \lambda)$ design is a pair $(V, \mathcal{B})$ in which $V$ is a set of cardinality $n$ and $\mathcal{B}$ is a collection of $k$-subsets of a point set $V$, such that every $t$-subset of $V$ is contained in exactly $\lambda$ elements of $\mathcal{B}$. Hence a $t$-subset-regular $k$-hypergraph $X$ of order $n$ is a $t-(n, k, \lambda)$ design in which $\lambda$ is equal to the $t$-valency of $X$. A large set of $t-(n, k, \lambda)$ designs of size $N$, denoted by $L S[N](t, k, n)$, is a partition of the complete design $\left(V, V^{(k)}\right)$ into $N$ disjoint $t-(n, k, \lambda)$ designs, where $\lambda=\left(\begin{array}{l}n-t \\ k-t\end{array}\right) / N$. If a $t$-subset regular $k$-hypergraph $X$ of order $n$ is self-complementary, then $X$ and its complement $X^{C}$ are both $t-(n, k, \lambda)$ designs with $\lambda=\left(\begin{array}{c}n-t \\ k-t\end{array}\right) / 2$. Hence the pair $\left\{X, X^{C}\right\}$ is an $L S[2](t, k, n)$ in which the $t$-designs are isomorphic. Hence results regarding sufficient conditions on the order a $t$-subset regular selfcomplementary $k$-hypergraph imply the corresponding results for the order of a $L S[2](t, k, n)$.

Theorem 1.1 was originally stated in the language of large sets of $t$-designs. Moreover, for $t \in\{1,2\}$, large sets $L S[2](t, k, n)$ have been constructed for all pairs of integer $n$ and $k$ satisfying condition (1) of Theorem 1.1 [1, 2, 3, 4, 5, 6, $7,8,14]$. However, it is important to note that these existence results do not imply that condition (1) of Theorem 1.1 is sufficient for $t \in\{1,2\}$, since there is no guarantee that two designs in the large sets constructed in these papers are isomorphic. To date, the only existence results for regular and 2-subset-regular self-complementary $k$-hypergraphs are those due to Rao, Potočnik, Šajna, and Knor $[10,11,13]$ mentioned in the last section.

In this paper, we will use terminology from hypergraph theory, rather than design theory.

\section{Necessary conditions on order}

Given the binary representation of a positive integer $k$, we can use Theorem 1.1 to obtain exact necessary conditions on the order of a $t$-subset-regular selfcomplementary $k$-hypergraph. We will denote the binary representation of an integer $k$ by the vector $b=\left(b_{m}, b_{m-1}, \ldots, b_{1}, b_{0}\right)_{2}$. This is, $b$ is the vector such that $k=\sum_{i=0}^{m} b_{i} 2^{i}, b_{m}=1$, and $b_{i} \in\{0,1\}$ for $0 \leq i \leq m$. The support of the binary representation $b$ is the set $\left\{i \in\{0,1,2, \ldots, m\}: b_{i}=1\right\}$, and is denoted by $\operatorname{supp}(b)$. 
Theorem 2.1. Let $k$ be a positive integer and let $b=\left(b_{m}, b_{m-1}, \ldots, b_{2}, b_{1}, b_{0}\right)_{2}$ be the binary representation of $k$. Let $t$ be an integer such that $1 \leq t<k$. If there exists a t-subset-regular self-complementary $k$-hypergraph, then

$$
n_{\left[2^{\ell+1}\right]} \in\left\{t, t+1, \ldots, k_{\left[2^{\ell+1}\right]}-1\right\}
$$

for some $\ell \in \operatorname{supp}(b)$.

Proof: By Theorem 1.1, there exists a positive integer $a$ such that

$$
n_{\left[2^{a}\right]} \in\left\{t, t+1, \ldots, k_{\left[2^{a}\right]}-1\right\} .
$$

If $a-1 \in \operatorname{supp}(b)$, then set $\ell=a-1$ and we are done. Hence we may assume that $a-1 \notin \operatorname{supp}(b)$.

If $i \notin \operatorname{supp}(b)$ for all $i$ such that $0 \leq i \leq a-1$, then $k_{\left[2^{a}\right]}=\sum_{i=0}^{a-1} b_{i} 2^{i}=0$, and so as $t \geq 1$, (4) implies that $n_{\left[2^{a}\right]} \in \emptyset$, giving a contradiction. Hence we must have $\operatorname{supp}(b) \cap\{1,2, \ldots, a-1\} \neq \emptyset$. Set

$$
\ell=\max (\operatorname{supp}(b) \cap\{1,2, \ldots, a-1\}) .
$$

Then $k_{\left[2^{a}\right]}=\sum_{i=0}^{a-1} b_{i} 2^{i}=\sum_{i=0}^{\ell} b_{i} 2^{i}=k_{\left[2^{\ell+1}\right]}$, and so (4) implies that

$$
n_{\left[2^{a}\right]} \in\left\{t, t+1, \ldots, k_{\left[2^{\ell+1}\right]}-1\right\} .
$$

Now (5) implies that $n_{\left[2^{a}\right]}<2^{\ell+1}$. Since $\ell+1<a$, it follows that $n_{\left[2^{\ell+1}\right]}=n_{\left[2^{a}\right]}$, and so (5) implies that

$$
n_{\left[2^{\ell+1}\right]} \in\left\{t, t+1, \ldots, k_{\left[2^{\ell+1}\right]}-1\right\} .
$$

Since $\ell \in \operatorname{supp}(b)$, this completes the proof.

In the case where $k$ is a sum of consecutive powers of 2, condition (3) of Theorem 2.1 holds for the largest integer in the support of the binary representation of $k$, as the next corollary shows.

Corollary 2.2. Let $r$ and $\ell$ be nonnegative integers, and suppose that $k=$ $\sum_{i=0}^{r} 2^{\ell+i}$. If there exists a t-subset-regular self-complementary $k$-hypergraph of order $n$, then $n_{\left[2^{\ell+r+1}\right]} \in\{t, t+1, \ldots, k-1\}$.

Proof: Let $b$ denote the binary representation of $k$. Then

$$
\operatorname{supp}(b)=\{\ell, \ell+1, \ldots, \ell+r\}
$$

and so Theorem 2.1 guarantees that

$$
n_{\left[2^{\ell+j+1}\right]} \in\left\{t, t+1, \ldots, k_{\left[2^{\ell+j+1}\right]}-1\right\}
$$

for some $j \in\{0,1, \ldots, r\}$. Suppose that $j<r$. Since

$$
n_{\left[2^{\ell+(j+1)+1}\right]} \leq 2^{\ell+j+1}+n_{\left[2^{\ell+j+1}\right]}
$$


and

$$
n_{\left[2^{\ell+(j+1)+1}\right]} \geq n_{\left[2^{\ell+j+1}\right]},
$$

(6) implies that

$$
t \leq n_{\left[2^{\ell+(j+1)+1}\right]}<2^{\ell+j+1}+k_{\left[2^{\ell+j+1}\right]} .
$$

Now since $2^{\ell+j+1}+k_{\left[2^{\ell+j+1}\right]}=2^{\ell+j+1}+\sum_{i=0}^{j} 2^{\ell+i}=k_{\left[2^{\ell+(j+1)+1}\right]}$, inequalities (7) imply that

$$
t \leq n_{\left[2^{\ell+(j+1)+1}\right]}<k_{\left[2^{\ell+(j+1)+1}\right]},
$$

and hence $n_{\left[2^{\ell+(j+1)+1}\right]} \in\left\{t, t+1, \ldots, k_{\left[2^{\ell+(j+1)+1}\right]}-1\right\}$. Thus for $j<r$, we have that

$$
\begin{aligned}
& n_{\left[2^{\ell+j+1}\right]} \in\left\{t, t+1, \ldots, k_{\left[2^{\ell+j+1}\right]}-1\right\} \\
\text { implies } & n_{\left[2^{\ell+(j+1)+1}\right]} \in\left\{t, t+1, \ldots, k_{\left[2^{\ell+(j+1)+1}\right]}-1\right\} .
\end{aligned}
$$

It follows that

$$
n_{\left[2^{\ell+r+1}\right]} \in\left\{t, t+1, \ldots, k_{\left[2^{\ell+r+1}\right]}-1\right\} .
$$

Since $k_{\left[2^{\ell+r+1}\right]}=k$, this implies that

$$
n_{\left[2^{\ell+r+1}\right]} \in\{t, t+1, \ldots, k-1\}
$$

as claimed.

Corollary 2.3. Let $\ell$ be a positive integer, let $k=2^{\ell}-1$ and let $t$ be a positive integer such that $t<k$. If there exists a $t$-subset-regular self-complementary $k$-hypergraph of order $n$, then $n_{\left[2^{\ell}\right]} \in\{t, t+1, \ldots, k-1\}$.

Proof: Since $k=2^{\ell}-1=\sum_{i=0}^{\ell-1} 2^{i}$, this result follows directly from Corollary 2.2 .

\section{Sufficient conditions on order}

In this section, we prove that a 1-subset-regular self-complementary $k$-hypergraph of order $n$ exists for every integer $n$ satisfying the necessary conditions of Theorem 1.1. Consequently, we prove Theorem 1.2. First we will need some notation.

Let $X=(\mathcal{V}, \mathcal{E})$ be a $k$-hypergraph and let $\theta \in \operatorname{Sym}(\mathcal{V})$. We will denote the valency $\operatorname{val}_{X}^{1}(\{v\})$ defined in Section 1.1 by $\operatorname{val}_{X}(v)$. The symbol $X^{\theta}$ denotes the hypergraph $\left(\mathcal{V}, \mathcal{E}^{\theta}\right)$, where $\mathcal{E}^{\theta}=\left\{E^{\theta}: E \in \mathcal{E}\right\}$ and $E^{\theta}=\left\{v^{\theta}: v \in E\right\}$. For a subset $\mathcal{P}$ of the orbits of $\theta$ on $\mathcal{V}^{(k)}$, let $\mathcal{U}(\mathcal{P})=\bigcup_{\mathcal{O} \in \mathcal{P}} \mathcal{O}$. For a subset $\mathcal{S} \subseteq \mathcal{V}^{(k)}$ and a vertex $v \in \mathcal{V}$, let $\operatorname{setval}_{\mathcal{S}}(v)$ denote the number of edges of $\mathcal{S}$ containing $v$.

We will often make use of the following lemma.

Lemma 3.1. Suppose that $X=(\mathcal{V}, \mathcal{E})$ is a self-complementary $k$-hypergraph. 
(1) $X$ is 1-subset-regular if and only if $v^{2} l_{X}(v)=v a l_{X^{C}}(v)$ for all $v \in \mathcal{V}$.

(2) If $\mathcal{V}=\{\infty\} \cup \mathbb{Z}_{n}$ and $\theta=(\infty)(01 \ldots(n-1)) \in \operatorname{Ant}(X)$ for an even positive integer $n$, then $X$ is 1-subset-regular if and only if $v^{2} l_{X}(0)=v_{a l} l_{X^{C}}(0)$.

\section{Proof:}

(1) If $\operatorname{val}_{X}(v)=\operatorname{val}_{X^{C}}(v)$, then

$$
\operatorname{val}_{X}(v)=\frac{1}{2}\left(\operatorname{val}_{X}(v)+\operatorname{val}_{X^{C}}(v)\right)=\frac{1}{2}\left(\operatorname{setval}_{\mathcal{V}^{(k)}}(v)\right)=\frac{1}{2}\left(\begin{array}{c}
|\mathcal{V}|-1 \\
k-1
\end{array}\right),
$$

which is independent of the choice of $v \in \mathcal{V}$. Thus $X$ is 1-subset-regular. Conversely, if $X$ is 1-subset-regular, then since $X \cong X^{C}$, the hypergraph $X^{C}$ is also 1-subset-regular and has the same 1-valency as $X$. Thus val $X(v)=$ $\operatorname{val}_{X^{C}}(v)$ for all $v \in \mathcal{V}$.

(2) If $X$ is 1 -subset-regular, then as $0 \in \mathcal{V}, \operatorname{val}_{X}(0)=\operatorname{val}_{X^{C}}(0)$ by part (1).

Conversely, suppose that $\operatorname{val}_{X}(0)=\operatorname{val}_{X^{C}}(0)$. Observe that for any orbit $\mathcal{O}$ of $\theta$ on $\mathcal{V}^{(k)}$, an element $x \in \mathbb{Z}_{n}$ lies in $c$ edges of $\mathcal{O} \cap \mathcal{E}$ if and only if $(x-1)_{[n]}$ lies in $c$ edges of $\mathcal{O} \cap \mathcal{E}^{C}$, which holds if and only if $(x-2)_{[n]}$ lies in $c$ edges of $\mathcal{O} \cap \mathcal{E}$. This implies that $\operatorname{val}_{X}(x)=\operatorname{val}_{X^{C}}(y)$ whenever $x \not \equiv y(\bmod 2)$, and $\operatorname{val}_{X}(x)=\operatorname{val}_{X}(y)$ whenever $x \equiv y(\bmod 2)$. Now since $\operatorname{val}_{X}(0)=\operatorname{val}_{X^{C}}(0)$, for $x$ even and $y$ odd, we have

$$
\operatorname{val}_{X}(x)=\operatorname{val}_{X}(0)=\operatorname{val}_{X^{C}}(0)=\operatorname{val}_{X}(y) .
$$

Hence $\operatorname{val}_{X}(x)=\operatorname{val}_{X}(y)$ for all $x, y \in \mathbb{Z}_{n}$. Moreover, since $\theta \in \operatorname{Ant}(X)$ and $\theta$ fixes $\infty$, we must also have $\operatorname{val}_{X}(\infty)=\operatorname{val}_{X^{C}}(\infty)$. Hence $\operatorname{val}_{X}(v)$ is independent of the choice of $v \in \mathcal{V}$, and so $X$ is 1-subset-regular.

In Lemma 3.4 we will prove that the necessary condition (1) in Theorem 1.1 on the order $n$ of a self-complementary $k$-hypergraph is sufficient by induction on the congruence class of $n$ modulo $2^{a}$. In Lemma 3.3 we will handle the base case where $n \equiv 1\left(\bmod 2^{a}\right)$, that is, $n=m 2^{a}+1$ for some positive integer $m$. We will need to make use of the following lemma, which handles the case where $m=1$.

For a positive integer $n$, a subset $A$ of $\mathbb{Z}_{n}$, and an element $b$ of $\mathbb{Z}_{n}$, let $A+b$ denote the set $\left\{(a+b)_{[n]}: a \in A\right\}$.

Lemma 3.2. Let $k$ and $a$ be positive integers such that $a \geq 2$ and $2 \leq k<2^{a}$. Let $\mathcal{V}=\{\infty\} \cup \mathbb{Z}_{2^{a}}$, where $\infty \notin \mathbb{Z}_{2^{a}}$. There exists a 1-subset-regular self-complementary $k$-hypergraph on $\mathcal{V}$ with antimorphism

$$
\theta=(\infty)\left(\begin{array}{lllll}
0 & 1 & 2 & \cdots & \left(2^{a}-1\right)
\end{array}\right) .
$$


Proof: First we will fix an integer $r$ such that $1 \leq r<2^{a}$ and examine the structure of the orbits of $\theta$ on $\mathbb{Z}_{2^{a}}^{(r)}$. In particular, we will examine how the number of even and odd elements of $E \in \mathbb{Z}_{2^{a}}^{(r)}$ affect the valency of 0 in the orbit of $\theta$ on $\mathbb{Z}_{2^{a}}^{(r)}$ containing $E$.

We can write $r=2^{z} M$ for some integer $z$ such that $0 \leq z \leq a-1$ and some odd positive integer $M$. Let $\mathcal{O}$ be an orbit of $\theta$ on $\mathbb{Z}_{2^{a}}^{(r)}$. Then $\mathcal{O}$ has length $2^{a-x}$ for some $x$ such that $0 \leq x \leq z$. For $x \in\{0,1, \ldots, z\}$, we will define a partition of the set $\left\{0,1,2, \ldots, 2^{a}-1\right\}$ into $2^{x}$ subsets $S_{0}^{x}, S_{1}^{x}, S_{2}^{x}, \ldots, S_{2^{x}-1}^{x}$ of consecutive integers, each of length $2^{a-x}$. For each $w \in\left\{0,1, \ldots, 2^{x}-1\right\}$, set $S_{w}^{x}:=\left\{w 2^{a-x}+v: v \in\left\{0,1, \ldots, 2^{a-x}-1\right\}\right\}$. Then $S_{w}^{x}=S_{0}^{x}+w 2^{a-x}$. If $\mathcal{O}$ has length $2^{a-x}$, any edge $E \in \mathcal{O}$ contains exactly $r / 2^{x}$ elements from each subset $S_{w}^{x}$ in the partition, and $E \cap S_{w}^{x}$ must be a translation of $E \cap S_{0}^{x}$, for all $w=0,1, \ldots, 2^{x}-1$. In particular $E \cap S_{w}^{x}=E \cap S_{0}^{x}+w 2^{a-x}$. Now if $E \cap S_{0}^{x}$ contains $i$ even elements and $j$ odd elements, then $E^{\theta} \cap S_{0}^{x}$ contains $j$ even elements and $i$ odd elements. Hence, for any orbit $\mathcal{O}$ of $\theta$ on $\mathbb{Z}_{2^{a}}^{(r)}$ of length $2^{a-x}$, there exist nonnegative integers $i$ and $j$ such that $i+j=r / 2^{x}$, and every edge of $\mathcal{O}$ contains exactly $i$ even elements and $j$ odd elements of $S_{0}^{x}$, or vice versa. Moreover, if $E \in \mathcal{O}$ and $E$ contains $i$ even and $j$ odd elements of $S_{0}^{x}$, then 0 lies in exactly $i$ elements of the sequence $E, E^{\theta^{2}}, E^{\theta^{4}}, \ldots, E^{\theta^{a^{a-x}-2}}$, and 0 lies in exactly $j=r / 2^{x}-i$ elements of the sequence $E^{\theta^{1}}, E^{\theta^{3}}, E^{\theta^{5}}, \ldots, E^{\theta^{2^{a-x}}-1}$.

For $x \in\{0,1, \ldots, z\}$ and $i \in\left\{0,1, \ldots, r / 2^{x+1}\right\}$, and $j=r / 2^{x}-i$, let $\mathcal{E}_{i, j}^{x}$ denote the set of orbits of $\theta$ on $\mathbb{Z}_{2^{a}}^{(r)}$ of length $2^{a-x}$ whose edges contain $i$ even and $j$ odd elements in the set $S_{0}^{x}$, or which contain $j$ even and $i$ odd elements of $S_{0}^{x}$. For each $\mathcal{O} \in \mathcal{E}_{i, j}^{x}$, choose an edge $E \in \mathcal{O}$. If the number of even elements of $E$ does not exceed the number of odd elements of $E$, colour the edges in the sequence $E, E^{\theta^{2}}, E^{\theta^{4}}, \ldots, E^{\theta^{2^{a-x}-2}}$ red and colour the edges in the sequence $E^{\theta^{1}}, E^{\theta^{3}}, E^{\theta^{5}}, \ldots, E^{{\theta^{2}-x}^{2-1}}$ blue. If $E$ has more even entries than odd entries, colour the edges in the sequence $E, E^{\theta^{2}}, E^{\theta^{4}}, \ldots, E^{\theta^{2^{a-x}-2}}$ blue and colour the edges in the sequence $E^{\theta^{1}}, E^{\theta^{3}}, E^{\theta^{5}}, \ldots, E^{\theta^{2^{a-x}-1}}$ red. For any subset $\mathcal{S}$ of $\mathbb{Z}_{2^{a}}^{(r)}$, let $\mathcal{S}_{\text {red }}$ and $\mathcal{S}_{\text {blue }}$ denote the set of red and blue edges in $\mathcal{S}$, respectively.

Since $i \leq j$, for each orbit $\mathcal{O} \in \mathcal{E}_{i, j}^{x}$ we have

$$
\operatorname{setval}_{\mathcal{O}_{\text {blue }}}(0)-\operatorname{setval}_{\mathcal{O}_{\text {red }}}(0)=j-i .
$$

Let $\hat{\mathcal{E}}_{r}$ be a subset of $\mathbb{Z}_{2^{a}}^{(r)}$ which contains the red edges from exactly $\left\lfloor\left|\mathcal{E}_{i, j}^{x}\right| / 2\right\rfloor$ orbits of $\mathcal{E}_{i, j}^{x}$, and the blue edges from the remaining orbits of $\mathcal{E}_{i, j}^{x}$, for all $0 \leq$ $x \leq z$, and for all $i, j$ such that $0 \leq i \leq r / 2^{x+1}$ and $j=r / 2^{x}-i$. If $\left|\mathcal{E}_{i, j}^{x}\right|$ is even, say $\left|\mathcal{E}_{i, j}^{x}\right|=2 \nu$ for a positive integer $\nu$, then

$$
\operatorname{setval}_{\mathcal{U}\left(\mathcal{E}_{i, j}^{x}\right) \cap \hat{\mathcal{E}}_{r}}(0)-\operatorname{setval}_{\mathcal{U}\left(\mathcal{E}_{i, j}^{x}\right) \cap \hat{\mathcal{E}}_{r}^{C}}(0)=(\nu i+\nu j)-(\nu i+\nu j)=0 .
$$


If $\left|\mathcal{E}_{i, j}^{x}\right|$ is odd, say $\left|\mathcal{E}_{i, j}^{x}\right|=2 \nu-1$ for a positive integer $\nu$, then

$$
\begin{aligned}
& \operatorname{setval}_{\mathcal{U}\left(\mathcal{E}_{i, j}^{x}\right) \cap \hat{\mathcal{E}}_{r}}(0)-\operatorname{setval}_{\mathcal{U}\left(\mathcal{E}_{i, j}^{x}\right) \cap \hat{\mathcal{E}}_{r}^{C}}(0) \\
= & ((\nu-1) i+\nu j)-(\nu i+(\nu-1) j) \\
= & j-i .
\end{aligned}
$$

If $x<z$, then $i+j=r / 2^{x}=2^{z-x} M$ is even, which implies that $j-i$ is even. On the other hand, if $x=z$, then $i+j=r / 2^{x}=r / 2^{z}=M$ is odd, which implies that $j-i$ is odd.

- Claim I: For a fixed integer $r=2^{z} M$ such that $M$ is odd and $1 \leq r<2^{a}$, the set $\mathcal{E}_{i, j}^{z}$ of orbits of $\theta$ on $\mathbb{Z}_{2^{a}}^{(r)}$ has odd cardinality for exactly one pair $i, j$ satisfying $0 \leq i<j$ and $i+j=M$.

- Proof of Claim I: First, note that the set $S_{0}^{z}$ contains exactly $2^{a-z-1}$ odd elements and $2^{a-z-1}$ even elements. Thus for $j>2^{a-z-1}$, we have $\mathcal{E}_{i, j}^{z}=\emptyset$, which has even cardinality. Hence we need only consider the case where $0 \leq i<j \leq 2^{a-z-1}$.

We will count the number of orbits in $\mathcal{E}_{i, j}^{z}$ where $i$ and $j$ are nonnegative integers such that $i<r / 2^{z+1}$ and $i+j=r / 2^{z}=M$. The number of ways to choose $i$ even elements and $j$ odd elements from the set $S_{0}^{z}$ is $\left(\begin{array}{c}2^{a-z-1} \\ i\end{array}\right)\left(\begin{array}{c}2^{a-z-1} \\ j\end{array}\right)$, which is also equal to the number of ways to choose $i$ odd elements and $j$ even elements from this set. Hence the number of edges which lie in $\mathcal{U}\left(\mathcal{E}_{i, j}^{z}\right)$ is $2\left({ }^{2^{a-z-1}}\right)\left({ }_{i}^{2^{a-z-1}}{ }_{j}\right)$. Since each orbit of $\mathcal{E}_{i, j}^{z}$ has length $2^{a-z}$, the number of orbits in $\mathcal{E}_{i, j}^{z}$ is

$$
\left|\mathcal{E}_{i, j}^{z}\right|=\frac{1}{2^{a-z-1}}\left(\begin{array}{c}
2^{a-z-1} \\
i
\end{array}\right)\left(\begin{array}{c}
2^{a-z-1} \\
j
\end{array}\right) \text {. }
$$

Case 1: $z=a-1$. In this case we have $r=2^{a-1} M<2^{a}$ for odd $M$, which implies that $M=1$ and $r=2^{a-1}$. Since $i+j=r / 2^{z}=2^{a-1} / 2^{a-1}=1$ and $i<j$, we must have $i=0$ and $j=1$, and so

$$
\left|\mathcal{E}_{i, j}^{z}\right|=\left|\mathcal{E}_{0,1}^{a-1}\right|=\frac{1}{2^{0}}\left(\begin{array}{c}
2^{0} \\
0
\end{array}\right)\left(\begin{array}{c}
2^{0} \\
1
\end{array}\right)=1
$$

which is odd.

Case 2: $z<a-1$. In this case, since $i+j=M$ is odd, and the cardinality in (8) is an integer, Lemma A.1 (see Appendix) implies that $\left|\mathcal{E}_{i, j}^{z}\right|$ is odd if and only if $i \in\left\{0,2^{a-z-1}\right\}$ or $j \in\left\{0,2^{a-z-1}\right\}$. We will show that exactly one of these situations occurs for $i<j$.

Since $0 \leq i<j \leq 2^{a-z-1}$, it follows that $j \neq 0$ and $i \neq 2^{a-z-1}$. Hence we need only check that exactly one of the conditions $i=0$ and $j=2^{a-z-1}$ hold. Since $z<a-1$, we must have $r \neq 2^{a-1}$. Suppose $r<2^{a-1}$. Then if 
$j=2^{a-z-1}$, we have $i=r / 2^{z}-j=r / 2^{z}-2^{a-z-1}<2^{a-z-1}-2^{a-z-1}=0$, contradicting the assumption that $i \geq 0$. However, there are edges such that $i=0$ and $j=r / 2^{z}<2^{a-1} / 2^{z}=2^{a-z-1}$. On the other hand, if $r>2^{a-1}$, then if $i=0$, we have $j=r / 2^{z}>2^{a-1} / 2^{z}=2^{a-z-1}$, and so $j>2^{a-z-1}$, giving a contradiction. However, there are edges such that $j=2^{a-z-1}$, for in this case $i=r / 2^{z}-j=r / 2^{z}-2^{a-z-1}>$ $2^{a-1} / 2^{z}-2^{a-z-1}=0$, so $0<i<2^{a-z-1}$.

We have shown that $j \neq 0, i \neq 2^{a-z-1}$, and that if $r<2^{a-1}$ then $j \neq 2^{a-z-1}$ but there exist orbits with $i=0$ and $j=r / 2^{z}<2^{a-z-1}$, and that if $r>2^{a-1}$ then $i \neq 0$ but there exist orbits with $j=2^{a-z-1}$ and $i=r / 2^{z}-j>0$.

Thus exactly one of the two situations $i=0$ and $j=2^{a-z-1}$ occurs for $i<j$, and neither of the two situations $i=2^{a-z-1}$ and $j=0$ can occur. Thus if $z<a-1$, exactly one of $\left|\mathcal{E}_{0, r / 2^{z}}^{z}\right|$ and $\left|\mathcal{E}_{\left(r / 2^{z}-2^{a-z-1}\right), 2^{a-z-1}}^{z}\right|$ is odd, and $\left|\mathcal{E}_{i, j}^{z}\right|$ is even for all other feasible pairs $i, j$. This completes the proof of Claim I.

Claim I and the comments preceding it imply that

$$
\operatorname{setval}_{\hat{\mathcal{E}}_{r}}(0)-\operatorname{setval}_{\hat{\mathcal{E}}_{r}^{C}}(0)
$$

is odd for all integers $r$ such that $1 \leq r<2^{a}$. Fix an integer $k$ such that $2 \leq k<2^{a}$. Then $1 \leq k-1<2^{a}$, and so

$$
\begin{aligned}
& \operatorname{setval}_{\hat{\mathcal{E}}_{k} \cup \hat{\mathcal{E}}_{k-1}}(0)-\operatorname{setval}_{\hat{\mathcal{E}}_{k}^{C} \cup \hat{\mathcal{E}}_{k-1}^{C}}(0) \\
= & \left(\operatorname{setval}_{\hat{\mathcal{E}}_{k}}(0)-\operatorname{setval}_{\hat{\mathcal{E}}_{k}^{C}}(0)\right)+\left(\operatorname{setval}_{\hat{\mathcal{E}}_{k-1}}(0)-\operatorname{setval}_{\hat{\mathcal{E}}_{k-1}^{C}}(0)\right)
\end{aligned}
$$

is even.

We will find subsets $\mathcal{E}_{k-1}^{\prime} \subseteq \mathbb{Z}_{2^{a}}^{(k-1)}$ and $\mathcal{E}_{k}^{\prime} \subseteq \mathbb{Z}_{2^{a}}^{(k)}$ which are related to $\hat{\mathcal{E}}_{k-1}$ and $\hat{\mathcal{E}_{k}}$, but for which the even quantity in $(9)$ is bounded. For each $r \in\{k-1, k\}$, if $r=2^{z} M$, then for all integers $x, i$ and $j$ such that $0 \leq x \leq z$, $0 \leq i \leq r / 2^{x+1}$, and $j=r / 2^{x}-i$, we define $\lambda^{r}(i, j, x)$ as

$$
\lambda^{r}(i, j, x)=\operatorname{setval}_{\mathcal{U}\left(\mathcal{E}_{i, j}^{x} \cap \hat{\mathcal{E}}_{r}\right)}(0)-\operatorname{setval}_{\mathcal{U}\left(\mathcal{E}_{i, j}^{x} \cap \hat{\mathcal{E}}_{r}^{C}\right)}(0)=j-i
$$

Note that $0 \leq j-i \leq r$. Thus setval $\hat{\mathcal{E}}_{r}(0)-\operatorname{setval}_{\hat{\mathcal{E}}_{r}^{C}}(0)$ is equal to the sum of a set $A_{r}$ of nonnegative integers for

$$
A_{r}=\left\{\lambda^{r}(i, j, x): 0 \leq x \leq z, 0 \leq i \leq r / 2^{x+1}, j=r / 2^{x}-i\right\},
$$

and each $\lambda \in A_{r}$ satisfies $0 \leq \lambda \leq r$. Hence Lemma A.2 (see Appendix) implies that there is a function $v: A_{r} \rightarrow\{-1,1\}$ such that $0 \leq \sum_{\lambda \in A_{r}} \lambda v(\lambda) \leq r$.

Form a subset $\mathcal{E}_{r}^{\prime}$ of $\mathbb{Z}_{2^{a}}^{(r)}$ from $\hat{\mathcal{E}}_{r}$ by swapping red edges for blue edges, and vice versa, in $\mathcal{E}_{i, j}^{x} \cap \hat{\mathcal{E}}_{r}$ whenever $v\left(\lambda^{r}(i, j, x)\right)=-1$. Then setval $\mathcal{E}_{r}^{\prime}(0)-$ 
$\operatorname{setval}_{\left(\mathcal{E}_{r}^{\prime}\right)^{C}}(0)$ has the same parity as setval $\hat{\mathcal{E}}_{r}(0)-\operatorname{setval}_{\hat{\mathcal{E}}_{r}^{C}}(0)$. Moreover,

$$
\operatorname{setval}_{\mathcal{E}_{r}^{\prime}}(0)-\operatorname{setval}_{\left(\mathcal{E}_{r}^{\prime}\right)^{C}}(0)=\sum_{\lambda \in A_{r}} \lambda v(\lambda)
$$

and so

$$
0 \leq \operatorname{setval}_{\mathcal{E}_{r}^{\prime}}(0)-\operatorname{setval}_{\left(\mathcal{E}_{r}^{\prime}\right)^{C}}(0) \leq r
$$

Thus

$$
\begin{aligned}
& \operatorname{setval}_{\mathcal{E}_{k}^{\prime} \cup \mathcal{E}_{k-1}^{\prime}}(0)-\operatorname{setval}_{\left(\mathcal{E}_{k}^{\prime}\right)^{C} \cup\left(\mathcal{E}_{k-1}^{\prime}\right)^{C}(0)} \\
& =\left(\operatorname{setval}_{\mathcal{E}_{k}^{\prime}}(0)-\operatorname{setval}_{\left(\mathcal{E}_{k}^{\prime}\right)^{C}}(0)\right)+\left(\operatorname{setval}_{\mathcal{E}_{k-1}^{\prime}}(0)-\operatorname{setval}_{\left(\mathcal{E}_{k-1}^{\prime}\right)^{C}}(0)\right)
\end{aligned}
$$

is equal to a nonnegative even number $2 \mu$ such that $2 \mu \leq 2 k-1$. But $2 \mu$ is even and $2 k-1$ is odd, so we must have $2 \mu \leq 2 k-2$, which implies that $0 \leq \mu \leq k-1$.

Case 1: $2 \leq k \leq 2^{a-1}$. Since $k$ or $k-1$ is even, it follows that $r-\mu$ is even for some $r \in\{k, k-1\}$. Fix this $r$. Then the system

$$
\begin{aligned}
-i+j & =\mu \\
i+j & =r
\end{aligned}
$$

has an integer solution $i=(r-\mu) / 2, j=(r+\mu) / 2$. Also, since $0 \leq \mu \leq r$, we are guaranteed that $0 \leq i, j \leq r$, and since $r \leq k \leq 2^{a-1}$ we also have $0 \leq i, j \leq 2^{a-1}$. For this $r$ there is an orbit $\mathcal{O} \in \mathcal{E}_{i, j}^{0}$ of $\theta$ on $\mathbb{Z}_{2^{a}}^{(r)}$ of full length $2^{a-0}$ such that $\mathcal{E}_{r}^{\prime}$ contains the red edges of $\mathcal{O}$, and

$$
\operatorname{setval}_{\mathcal{O}_{\text {blue }}}(0)-\operatorname{setval}_{\mathcal{O}_{\text {red }}}(0)=j-i=\mu .
$$

Let $\mathcal{E}_{k-1} \cup \mathcal{E}_{k}$ be the set of edges in $\mathbb{Z}_{2^{a}}^{(k-1)} \cup \mathbb{Z}_{2^{a}}^{(k)}$ obtained from $\mathcal{E}_{k-1}^{\prime} \cup \mathcal{E}_{k}^{\prime}$ by swapping red edges for blue edges in the orbit $\mathcal{O}$. Then (10) implies that

$$
\begin{aligned}
& \operatorname{setval}_{\mathcal{E}_{k} \cup \mathcal{E}_{k-1}}(0)-\operatorname{setval}_{\mathcal{E}_{k}^{C} \cup \mathcal{E}_{k-1}^{C}}(0) \\
& =\left(\operatorname{setval}_{\mathcal{E}_{k}^{\prime} \cup \mathcal{E}_{k-1}^{\prime}}(0)-\operatorname{setval}_{\left(\mathcal{E}_{k}^{\prime}\right)^{C} \cup\left(\mathcal{E}_{k-1}^{\prime}\right)^{C}}(0)\right)-2 \mu \\
& =2 \mu-2 \mu=0 .
\end{aligned}
$$

Finally, define $\mathcal{X}_{k}$ to be the hypergraph with vertex set $\mathcal{V}=\mathbb{Z}_{2^{a}} \cup\{\infty\}$ and edge set $\mathcal{E}=\mathcal{E}_{k} \cup\left\{E \cup\{\infty\}: E \in \mathcal{E}_{k-1}\right\}$. Since $\theta$ maps red edges onto blue edges within each orbit, and vice versa, it follows that $\theta \in \operatorname{Ant}\left(\mathcal{X}_{k}\right)$, and so $\mathcal{X}_{k}$ is self-complementary. Moreover, (11) implies that val $\mathcal{X}_{k}(0)=\operatorname{val}_{\mathcal{X}_{k}^{C}}(0)$, and so Lemma 3.1(2) guarantees that $\mathcal{X}_{k}$ is 1-subset-regular.

Case 2: $2^{a-1}<k<2^{a}$. In this case $2 \leq \hat{k}=2^{a}-(k-1) \leq 2^{a-1}$ and so by Case 1 there exists a 1 -subset-regular self-complementary $\hat{k}$-hypergraph 
$\mathcal{X}_{\hat{k}}$ on $\mathcal{V}$ with antimorphism $\theta$. Let $\mathcal{F}_{\hat{k}}$ denote the set of edges of $\mathcal{X}_{\hat{k}}$ which do not contain $\infty$, and let

$$
\mathcal{F}_{\hat{k}-1}=\left\{E \backslash\{\infty\}: E \in \mathcal{E}\left(\mathcal{X}_{\hat{k}}\right), \infty \in E\right\} .
$$

Since $\mathcal{X}_{\hat{k}}$ is 1 -subset-regular and self-complementary, it follows that

$$
\operatorname{setval}_{\mathcal{F}_{\hat{k}} \cup \mathcal{F}_{\hat{k}-1}}(0)=\operatorname{setval}_{\mathcal{F}_{\hat{k}}^{C} \cup \mathcal{F}_{\hat{k}-1}^{C}}(0) .
$$

Let

$$
\mathcal{E}_{k-1}=\left\{\mathbb{Z}_{2^{a}} \backslash E: E \in \mathcal{F}_{\hat{k}}\right\}
$$

and

$$
\mathcal{E}_{k}=\left\{\mathbb{Z}_{2^{a}} \backslash E: E \in \mathcal{F}_{\hat{k}-1}\right\} .
$$

Then $\mathcal{E}_{k-1} \subset \mathbb{Z}_{2^{a}}^{(k-1)}$ and $\mathcal{E}_{k} \subset \mathbb{Z}_{2^{a}}^{(k)}$. Moreover, (12) implies that

$$
\operatorname{setval}_{\mathcal{E}_{k} \cup \mathcal{E}_{k-1}}(0)=\operatorname{setval}_{\mathcal{E}_{k}^{C} \cup \mathcal{E}_{k-1}^{C}}(0) .
$$

Define $\mathcal{X}_{k}$ to be the hypergraph with vertex set $\mathcal{V}=\mathbb{Z}_{2^{a}} \cup\{\infty\}$ and edge set $\mathcal{E}=\mathcal{E}_{k} \cup\left\{E \cup\{\infty\}: E \in \mathcal{E}_{k-1}\right\}$. Then $\mathcal{X}_{k}$ is a $k$-hypergraph on $\mathcal{V}$, and since $\theta \in \operatorname{Ant}\left(\mathcal{X}_{\hat{k}}\right)$ it follows that $\theta \in \operatorname{Ant}\left(\mathcal{X}_{k}\right)$, and so $\mathcal{X}_{k}$ is self-complementary. Moreover, (13) implies that val $\mathcal{X}_{k}(0)=\operatorname{val}_{\mathcal{X}_{k}^{C}}(0)$, and so Lemma 3.1(2) guarantees that $\mathcal{X}_{k}$ is 1 -subset-regular, as required.

We are on our way to proving the sufficiency of condition (1) in the main result of this section, Theorem 1.2. In the next lemma, we state and prove the base case for the inductive proof of this sufficiency, which is given in Lemma 3.4.

Lemma 3.3. Let $a, k$, and $m$ be positive integers such that $a \geq 2$ and $k_{\left[2^{a}\right]} \geq 2$. Let $\mathcal{R}=\mathbb{Z}_{m 2^{a}}$, and let $\mathcal{V}=\{\infty\} \cup \mathcal{R}$. There exists a 1-subset-regular self-complementary $k$-hypergraph on $\mathcal{V}$ with antimorphism

$$
\theta=(\infty) \prod_{j=0}^{m-1}\left(j 2^{a}, j 2^{a}+1, \ldots,(j+1) 2^{a}-1\right) .
$$

Proof: We will construct a 1-subset-regular self-complementary $k$-hypergraph $\mathcal{Y}_{k}$ on $\mathcal{V}$ with antimorphism $\theta$.

For each $j \in \mathbb{Z}_{m}$, let

$$
\mathcal{R}_{j}=\left\{j 2^{a}, j 2^{a}+1, \ldots,(j+1) 2^{a}-1\right\},
$$

and let

$$
\theta_{j}=(\infty)\left(j 2^{a}, j 2^{a}+1, \ldots,(j+1) 2^{a}-1\right) \in \operatorname{Sym}\left(\mathcal{R}_{j} \cup\{\infty\}\right) .
$$

By Lemma 3.2, there exists a 1-subset-regular self-complementary $r$-hypergraph $\mathcal{X}_{r}^{j}$ on $\{\infty\} \cup \mathcal{R}_{j}$, with antimorphism $\theta_{j}$, for $r \in\left\{2,3, \ldots, 2^{a}-1\right\}$. 
For each $E \in \mathcal{V}^{(k)}$, let $C_{1}(E)=\left\{j \in \mathbb{Z}_{m}: 2 \leq\left|\left(\{\infty\} \cup \mathcal{R}_{j}\right) \cap E\right| \leq 2^{a}-1\right\}$. If $C_{1}(E) \neq \emptyset$, set $j_{1}(E)=\min \left\{j: j \in C_{1}(E)\right\}$. If $E \in \mathcal{V}^{(k)}$ and $C_{1}(E)=\emptyset$, then $\left|\left(\{\infty\} \cup \mathcal{R}_{j}\right) \cap E\right| \leq 1$ or $\left|\left(\{\infty\} \cup \mathcal{R}_{j}\right) \cap E\right| \geq 2^{a}$ for all $j \in \mathbb{Z}_{m}$. Since $2 \leq k_{\left[2^{a}\right]}<2^{a}$, this implies that one of the following conditions hold when $C_{1}(E)=\emptyset$ :

- $\infty \notin E$, all cycles of $\theta$ contain exactly 0,1 , or $2^{a}$ elements of $E$, and at least two nontrivial cycles of $\theta$ contain exactly one element of $E$.

- $\infty \in E$, all nontrivial cycles of $\theta$ contain at least $2^{a}-1$ elements of $E$, and at least two nontrivial cycles of $\theta$ contain exactly $2^{a}-1$ elements of $E$.

For each $E \in \mathcal{V}^{(k)}$ with $C_{1}(E)=\emptyset$, define $C_{2}(E)=\left\{j \in \mathbb{Z}_{m}:\left|\left(E \cap \mathcal{R}_{j}\right)\right| \in\right.$ $\left.\left\{1,2^{a}-1\right\}\right\}$. Then $\left|C_{2}(E)\right| \geq 2$. Let $i_{1}(E)$ and $i_{2}(E)$ be the two smallest elements of $C_{2}(E)$.

Define $\mathcal{Y}_{k}$ to be the $k$-hypergraph with vertex set $\mathcal{V}$ and edge set $\mathcal{E}$ such that an element $E \in \mathcal{V}^{(k)}$ is in $\mathcal{E}$ if and only if one of the following conditions hold for $j_{1}=j_{1}(E), i_{1}=i_{1}(E)$, and $i_{2}=i_{2}(E)$.

(i) $C_{1}(E) \neq \emptyset,\left|E \cap\left(\{\infty\} \cup \mathcal{R}_{j_{1}}\right)\right|=r$, and $E \cap\left(\{\infty\} \cup \mathcal{R}_{j_{1}}\right) \in \mathcal{E}\left(\mathcal{X}_{r}^{j_{1}}\right)$.

(ii) $C_{1}(E)=\emptyset, \infty \notin E, E \cap \mathcal{R}_{i_{1}}=\{x\}, E \cap \mathcal{R}_{i_{2}}=\{y\}$, and $(x+y)_{[4]} \in\{1,2\}$.

(iii) $C_{1}(E)=\emptyset, \infty \in E, \mathcal{R}_{i_{1}} \backslash E=\{x\}, \mathcal{R}_{i_{2}} \backslash E=\{y\}$, and $(x+y)_{[4]} \in\{1,2\}$.

We will prove that $\mathcal{Y}_{k}$ is 1 -subset-regular and self-complementary with antimorphism $\theta$.

First we will show that $\mathcal{Y}_{k}$ is self-complementary. Note that $\mathcal{E}^{C}=\mathcal{V}^{(k)} \backslash \mathcal{E}$ is the set of elements $E$ of $\mathcal{V}^{(k)}$ for which one of the following conditions hold. (Again, $j_{1}=j_{1}(E), i_{1}=i_{1}(E)$, and $i_{2}=i_{2}(E)$.)

(i) ${ }^{\prime} C_{1}(E) \neq \emptyset,\left|E \cap\left(\{\infty\} \cup \mathcal{R}_{j_{1}}\right)\right|=r$, and $E \cap\left(\{\infty\} \cup \mathcal{R}_{j_{1}}\right) \notin \mathcal{E}\left(\mathcal{X}_{r}^{j_{1}}\right)$.

(ii) ${ }^{\prime} C_{1}(E)=\emptyset, \infty \notin E, E \cap \mathcal{R}_{i_{1}}=\{x\}, E \cap \mathcal{R}_{i_{2}}=\{y\},(x+y)_{[4]} \in\{0,3\}$.

$(\text { iii })^{\prime} C_{1}(E)=\emptyset, \infty \in E, \mathcal{R}_{i_{1}} \backslash E=\{x\}, \mathcal{R}_{i_{2}} \backslash E=\{y\}$, and $(x+y)_{[4]} \in\{0,3\}$.

Observe that $\left.\theta\right|_{\{\infty\} \cup \mathcal{R}_{j_{1}}}=\theta_{j_{1}} \in \operatorname{Ant}\left(\mathcal{X}_{r}^{j_{1}}\right)$. Hence an element $E \in \mathcal{V}^{(k)}$ satisfies condition (i) if and only if $E^{\theta}$ satisfies condition (i)'. Also, for $x \in R_{i_{1}}, y \in R_{i_{2}}$, and $a \geq 2$, we have $\left(x^{\theta}+y^{\theta}\right)_{[4]}=\left((x+1)_{\left[2^{a}\right]}+(y+1)_{\left[2^{a}\right]}\right)_{[4]}=(x+y+2)_{[4]}$, so $\theta$ maps elements $x$ and $y$ with $(x, y) \in \mathcal{R}_{i_{1}} \times \mathcal{R}_{i_{2}}$ and $(x+y)_{[4]} \in\{1,2\}$ to elements $x^{\theta}$ and $y^{\theta}$ with $\left(x^{\theta}, y^{\theta}\right) \in \mathcal{R}_{i_{1}} \times \mathcal{R}_{i_{2}}$ and $\left(x^{\theta}+y^{\theta}\right)_{[4]} \in\{0,3\}$, and vice versa. It follows that an element $E \in \mathcal{V}^{(k)}$ satisfies condition (ii) if and only if $E^{\theta}$ satisfies condition (ii) ${ }^{\prime}$, and $E$ satisfies condition (iii) if and only if $E^{\theta}$ satisfies condition (iii) $)^{\prime}$ Hence $E \in \mathcal{E}$ if and only if $E^{\theta} \in \mathcal{E}^{C}$. Thus $\theta \in \operatorname{Ant}\left(\mathcal{Y}_{k}\right)$ and $\mathcal{Y}_{k}$ is self-complementary.

Next we show that $\mathcal{Y}_{k}$ is 1 -subset-regular, which by Lemma 3.1(1) is true if and only if $\operatorname{val}_{\mathcal{Y}_{k}}(v)=\operatorname{val}_{\mathcal{Y}_{k}^{C}}(v)$ for all $v \in \mathcal{V}$. Since $\theta \in \operatorname{Ant}\left(\mathcal{Y}_{k}\right)$ and $\theta$ 
fixes $\infty$, we certainly have $\operatorname{val}_{\mathcal{Y}_{k}}(\infty)=\operatorname{val}_{\mathcal{Y}_{k}^{C}}(\infty)$. It remains to show that $\operatorname{val}_{\mathcal{Y}_{k}}(v)=\operatorname{val}_{\mathcal{Y}_{k}^{C}}(v)$ for all $v \in \mathcal{R}$.

Let $j^{\prime} \in \mathbb{Z}_{m}$ and suppose that $v \in \mathcal{R}_{j^{\prime}}$. Let $\mathcal{O}$ be an orbit of $\theta$ on $\mathcal{V}^{(k)}$ which contains edges containing $v$. Let $E \in \mathcal{O}$, and set $C_{1}(\mathcal{O})=C_{1}(E)$, and if $C_{1}(E) \neq \emptyset$, set $j_{1}(\mathcal{O})=j_{1}(E)$. Note that $C_{1}(E)$ is constant over all $E \in \mathcal{O}$, and so $C_{1}(\mathcal{O})$ is independent of our choice of $E \in \mathcal{O}$, and so is $j_{1}(\mathcal{O})$, if it exists. If $C_{1}(\mathcal{O})=\emptyset$, set $C_{2}(\mathcal{O})=C_{2}(E)$, and set $i_{1}(\mathcal{O})=i_{1}(E)$ and $i_{2}(\mathcal{O})=i_{2}(E)$. If $C_{1}(\mathcal{O})=\emptyset$, then $C_{2}(\mathcal{O})$ is constant over all $E \in \mathcal{O}$, and so $C_{2}(\mathcal{O}), i_{1}(\mathcal{O})$, and $i_{2}(\mathcal{O})$ are also independent of our choice of $E$. Now $\mathcal{O}$ is one of four types:

- TYPE 1: $C_{1}(\mathcal{O}) \neq \emptyset$ and $j^{\prime} \neq j_{1}(\mathcal{O})$.

- TYPE 2: $C_{1}(\mathcal{O}) \neq \emptyset$ and $j^{\prime}=j_{1}(\mathcal{O})$.

- TYPE 3: $C_{1}(\mathcal{O})=\emptyset$ and $j^{\prime} \notin\left\{i_{1}(\mathcal{O}), i_{2}(\mathcal{O})\right\}$.

- TYPE 4: $C_{1}(\mathcal{O})=\emptyset$ and $j^{\prime} \in\left\{i_{1}(\mathcal{O}), i_{2}(\mathcal{O})\right\}$.

For each $i \in\{1,2,3,4\}$, let $\mathcal{P}_{i}$ be the set of orbits of $\theta$ on $\mathcal{V}^{(k)}$ of TYPE $i$ which contain edges containing $v$. We will show that

$$
\operatorname{setval}_{\mathcal{U}\left(\mathcal{P}_{i}\right) \cap \mathcal{E}}(v)=\operatorname{setval}_{\mathcal{U}\left(\mathcal{P}_{i}\right) \cap \mathcal{E}^{C}}(v)
$$

for all $i \in\{1,2,3,4\}$. For each $i$, let $\left(\mathcal{U}\left(\mathcal{P}_{i}\right) \cap \mathcal{E}\right)_{v}=\left\{E \in \mathcal{U}\left(\mathcal{P}_{i}\right) \cap \mathcal{E}: v \in E\right\}$, and let $\left(\mathcal{U}\left(P_{i}\right) \cap \mathcal{E}^{C}\right)_{v}=\left\{E \in \mathcal{U}\left(\mathcal{P}_{i}\right) \cap \mathcal{E}^{C}: v \in E\right\}$.

First consider the orbits of $\mathcal{P}_{1}$. Define the mapping $\beta_{1}:\left(\mathcal{U}\left(\mathcal{P}_{1}\right) \cap \mathcal{E}\right)_{v} \rightarrow$ $\left(\mathcal{U}\left(\mathcal{P}_{1}\right) \cap \mathcal{E}^{C}\right)_{v}$ by

$$
E^{\beta_{1}}=\left(\mathcal{R}_{j_{1}} \cap E^{\theta}\right) \cup\left(E \backslash \mathcal{R}_{j_{1}}\right),
$$

for all $E \in\left(\mathcal{U}\left(\mathcal{P}_{1}\right) \cap \mathcal{E}\right)_{v}$, where $j_{1}=j_{1}(\mathcal{O})$ for the orbit $\mathcal{O}$ of $\theta$ on $\mathcal{V}^{(k)}$ containing E.

Since $j^{\prime} \neq j_{1}$ for all orbits $\mathcal{O} \in \mathcal{P}_{1}$, and since $v \in \mathcal{R}_{j^{\prime}}$, it follows that for all $E \in\left(\mathcal{U}\left(\mathcal{P}_{1}\right) \cap \mathcal{E}\right)_{v}$ we have $v \in E \backslash \mathcal{R}_{j_{1}}$. Hence $\beta_{1}$ maps edges of $\left(\mathcal{U}\left(\mathcal{P}_{1}\right) \cap \mathcal{E}\right)_{v}$ to edges of $\left(\mathcal{U}\left(\mathcal{P}_{1}\right) \cap \mathcal{E}^{C}\right)_{v}$. Moreover, one can verify that $\beta_{1}$ is invertible, with inverse $\beta_{1}^{-1}$ defined by

$$
E^{\beta_{1}^{-1}}=\left(\mathcal{R}_{j_{1}} \cap E^{\theta^{-1}}\right) \cup\left(E \backslash \mathcal{R}_{j_{1}}\right),
$$

for all $E \in\left(\mathcal{U}\left(\mathcal{P}_{1}\right) \cap \mathcal{E}^{C}\right)_{v}$, where $j_{1}=j_{1}(\mathcal{O})$ for the orbit $\mathcal{O}$ of $\theta$ on $\mathcal{V}^{(k)}$ containing $E$. We conclude that $\left|\left(\mathcal{U}\left(\mathcal{P}_{1}\right) \cap \mathcal{E}\right)_{v}\right|=\left|\left(\mathcal{U}\left(\mathcal{P}_{1}\right) \cap \mathcal{E}^{C}\right)_{v}\right|$, and hence

$$
\operatorname{setval}_{\mathcal{U}\left(\mathcal{P}_{1}\right) \cap \mathcal{E}}(v)=\operatorname{setval}_{\mathcal{U}\left(\mathcal{P}_{1}\right) \cap \mathcal{E}^{C}}(v) .
$$

Next consider the orbits of $\mathcal{P}_{2}$. Every orbit $\mathcal{O}$ of $\mathcal{P}_{2}$ satisfies $j_{1}(\mathcal{O})=j^{\prime}$, and so $E \cap\left(\{\infty\} \cup \mathcal{R}_{j_{1}}\right) \in \mathcal{E}\left(\mathcal{X}_{r}^{j_{1}}\right)$, where $r=\left|E \cap\left(\{\infty\} \cup \mathcal{R}_{j_{1}}\right)\right|$, for all $E \in \mathcal{O} \cap \mathcal{E}$. Observe that since $\mathcal{X}_{r}^{j_{1}}$ is 1 -subset-regular and self-complementary for all $r$, by Lemma 3.1(1) we have

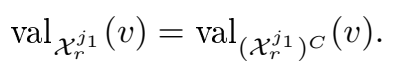


This implies that there is a bijection $\delta$ between the set of edges of $\mathcal{X}_{r}^{j_{1}}$ containing $v$ and the set of edges of $\left(\mathcal{X}_{r}^{j_{1}}\right)^{C}$ containing $v$. Now define the mapping $\beta_{2}$ : $\left(\mathcal{U}\left(\mathcal{P}_{2}\right) \cap \mathcal{E}\right)_{v} \rightarrow\left(\mathcal{U}\left(\mathcal{P}_{2}\right) \cap \mathcal{E}^{C}\right)_{v}$ by

$$
E^{\beta_{2}}=\left(E \cap\left(\{\infty\} \cup \mathcal{R}_{j_{1}}\right)\right)^{\delta} \cup\left(E \backslash\left(\{\infty\} \cup \mathcal{R}_{j_{1}}\right)\right),
$$

for all $E \in\left(\mathcal{U}\left(\mathcal{P}_{2}\right) \cap \mathcal{E}\right)_{v}$, where $j_{1}=j_{1}(\mathcal{O})$ for the orbit $\mathcal{O}$ of $\theta$ on $\mathcal{V}^{(k)}$ containing $E$.

Since $j^{\prime}=j_{1}$ for all orbits $\mathcal{O} \in \mathcal{P}_{2}$, and $v \in \mathcal{R}_{j_{1}}$, the definition of $\delta$ guarantees that $v \in E \cap\left(\{\infty\} \cup \mathcal{R}_{j_{1}}\right)$ if and only if $v \in\left(E \cap\left(\{\infty\} \cup \mathcal{R}_{j_{1}}\right)\right)^{\delta}$. Also, condition (i) guarantees that $E \in \mathcal{U}\left(\mathcal{P}_{2}\right) \cap \mathcal{E}$ if and only if $E^{\beta_{2}} \in \mathcal{U}\left(\mathcal{P}_{2}\right) \cap \mathcal{E}^{C}$. Hence $\beta_{2}$ maps edges of $\left(\mathcal{U}\left(\mathcal{P}_{2}\right) \cap \mathcal{E}\right)_{v}$ to edges of $\left(\mathcal{U}\left(\mathcal{P}_{2}\right) \cap \mathcal{E}^{C}\right)_{v}$. Moreover, one can verify that $\beta_{2}$ is invertible, with inverse $\beta_{2}^{-1}$ defined by

$$
E^{\beta_{2}^{-1}}=\left(E \cap\left(\{\infty\} \cup \mathcal{R}_{j_{1}}\right)\right)^{\delta^{-1}} \cup\left(E \backslash\left(\{\infty\} \cup \mathcal{R}_{j_{1}}\right)\right),
$$

for all $E \in\left(\mathcal{U}\left(\mathcal{P}_{2}\right) \cap \mathcal{E}^{C}\right)_{v}$, where $j_{1}=j_{1}(\mathcal{O})$ for the orbit $\mathcal{O}$ of $\theta$ on $\mathcal{V}^{(k)}$ containing $E$. We conclude that $\left|\left(\mathcal{U}\left(\mathcal{P}_{2}\right) \cap \mathcal{E}\right)_{v}\right|=\left|\left(\mathcal{U}\left(\mathcal{P}_{2}\right) \cap \mathcal{E}^{C}\right)_{v}\right|$, and hence

$$
\operatorname{setval}_{\mathcal{U}\left(\mathcal{P}_{2}\right) \cap \mathcal{E}}(v)=\operatorname{setval}_{\mathcal{U}\left(\mathcal{P}_{2}\right) \cap \mathcal{E}^{C}}(v) .
$$

Now consider the orbits of $\mathcal{P}_{3}$. Define the mapping $\beta_{3}:\left(\mathcal{U}\left(\mathcal{P}_{3}\right) \cap \mathcal{E}\right)_{v} \rightarrow$ $\left(\mathcal{U}\left(\mathcal{P}_{3}\right) \cap \mathcal{E}^{C}\right)_{v}$ by

$$
E^{\beta_{3}}=\left(\left(\mathcal{R}_{i_{1}} \cup \mathcal{R}_{i_{2}}\right) \cap E^{\theta}\right) \cup\left(E \backslash\left(\mathcal{R}_{i_{1}} \cup \mathcal{R}_{i_{2}}\right)\right),
$$

for all $E \in\left(\mathcal{U}\left(\mathcal{P}_{3}\right) \cap \mathcal{E}\right)_{v}$, where $i_{1}=i_{1}(\mathcal{O})$ and $i_{2}=i_{2}(\mathcal{O})$ for the orbit $\mathcal{O}$ of $\theta$ on $\mathcal{V}^{(k)}$ containing $E$.

Since $j^{\prime} \notin\left\{i_{1}, i_{2}\right\}$ for all orbits $\mathcal{O} \in \mathcal{P}_{3}$, and $v \in \mathcal{R}_{j^{\prime}}$, for all $E \in\left(\mathcal{U}\left(\mathcal{P}_{3}\right) \cap \mathcal{E}\right)_{v}$ we have $v \in E \backslash\left(\mathcal{R}_{i_{1}} \cup \mathcal{R}_{i_{2}}\right)$. Hence $\beta_{3}$ maps edges of $\left(\mathcal{U}\left(\mathcal{P}_{3}\right) \cap \mathcal{E}\right)_{v}$ to edges of $\left(\mathcal{U}\left(\mathcal{P}_{3}\right) \cap \mathcal{E}^{C}\right)_{v}$. Moreover, one can verify that $\beta_{3}$ is invertible, with inverse $\beta_{3}^{-1}$ defined by

$$
E^{\beta_{3}^{-1}}=\left(\left(\mathcal{R}_{i_{1}} \cup \mathcal{R}_{i_{2}}\right) \cap E^{\theta^{-1}}\right) \cup\left(E \backslash\left(\mathcal{R}_{i_{1}} \cup \mathcal{R}_{i_{2}}\right)\right),
$$

for all $E \in\left(\mathcal{U}\left(\mathcal{P}_{3}\right) \cap \mathcal{E}^{C}\right)_{v}$, where $i_{1}=i_{1}(\mathcal{O})$ and $i_{2}=i_{2}(\mathcal{O})$ for the orbit $\mathcal{O}$ of $\theta$ on $\mathcal{V}^{(k)}$ containing $E$. We conclude that $\left|\left(\mathcal{U}\left(\mathcal{P}_{3}\right) \cap \mathcal{E}\right)_{v}\right|=\left|\left(\mathcal{U}\left(\mathcal{P}_{3}\right) \cap \mathcal{E}^{C}\right)_{v}\right|$, and hence

$$
\operatorname{setval}_{\mathcal{U}\left(\mathcal{P}_{3}\right) \cap \mathcal{E}}(v)=\operatorname{setval}_{\mathcal{U}\left(\mathcal{P}_{3}\right) \cap \mathcal{E}^{C}}(v) .
$$

Finally, consider the orbits of $\mathcal{P}_{4}$. Every orbit $\mathcal{O}$ of $\mathcal{P}_{4}$ satisfies $j^{\prime} \in\left\{i_{1}, i_{2}\right\}$. Since $v \in \mathcal{R}_{j^{\prime}}$, we must have $v \in \mathcal{R}_{i_{1}} \cup \mathcal{R}_{i_{2}}$. Assume, without loss of generality, that $v \in \mathcal{R}_{i_{1}}$. Define the mapping $\beta_{4}:\left(\mathcal{U}\left(\mathcal{P}_{4}\right) \cap \mathcal{E}\right)_{v} \rightarrow\left(\mathcal{U}\left(\mathcal{P}_{4}\right) \cap \mathcal{E}^{C}\right)_{v}$ by

$$
E^{\beta_{4}}=\left(\mathcal{R}_{i_{2}} \cap E^{\theta^{2}}\right) \cup\left(E \backslash \mathcal{R}_{i_{2}}\right),
$$

for all $E \in\left(\mathcal{U}\left(\mathcal{P}_{4}\right) \cap \mathcal{E}\right)_{v}$, where in each case $i_{1}=i_{1}(\mathcal{O})$ and $i_{2}=i_{2}(\mathcal{O})$ for the orbit $\mathcal{O}$ of $\theta$ on $\mathcal{V}^{(k)}$ containing $E$. Now since $v \in \mathcal{R}_{i_{1}}$, it follows that $v \in E \backslash \mathcal{R}_{i_{2}}$ 
for all $E$ in $\left(\mathcal{U}\left(\mathcal{P}_{4}\right) \cap \mathcal{E}\right)_{v}$. Now observe that if $E \in\left(\mathcal{U}\left(\mathcal{P}_{4}\right) \cap \mathcal{E}\right)_{v}$, then either $\left|E \cap \mathcal{R}_{i_{1}}\right|=\left|E \cap \mathcal{R}_{i_{2}}\right|=1$ or $\left|\mathcal{R}_{i_{1}} \backslash E\right|=\left|\mathcal{R}_{i_{2}} \backslash E\right|=1$. In the former case, we must have $E \cap \mathcal{R}_{i_{1}}=\{v\}$ and $E \cap \mathcal{R}_{i_{2}}=\{w\}$, for some $w \in \mathcal{R}_{i_{2}}$ such that $(v+w)_{[4]} \in\{1,2\}$, which implies that $E^{\beta_{4}} \cap \mathcal{R}_{i_{1}}=\{v\}, E^{\beta_{4}} \cap \mathcal{R}_{i_{2}}=\left\{(w+2)_{\left[2^{a}\right]}\right\}$, and $\left(v+(w+2)_{\left[2^{a}\right]}\right)_{[4]}=(v+w+2)_{[4]} \in\{0,3\}$, since $a \geq 2$. In the latter case, we must have $\mathcal{R}_{i_{1}} \backslash E=\{x\}$ and $\mathcal{R}_{i_{2}} \backslash E=\{y\}$, for some $x \in \mathcal{R}_{i_{1}}$ and $y \in \mathcal{R}_{i_{2}}$ such that $x \neq v$ and $(x+y)_{[4]} \in\{1,2\}$, which implies that $\mathcal{R}_{i_{1}} \backslash E^{\beta_{4}}=\{x\}$, $\mathcal{R}_{i_{2}} \backslash E^{\beta_{4}}=\left\{(y+2)_{\left[2^{a}\right]}\right\}$, and $\left(x+(y+2)_{\left[2^{a}\right]}\right)_{[4]}=(x+y+2)_{[4]} \in\{0,3\}$, since $a \geq 2$. Hence conditions (ii) and (iii) guarantee that $\beta_{4}$ maps edges of $\left(\mathcal{U}\left(\mathcal{P}_{4}\right) \cap \mathcal{E}\right)_{v}$ to edges of $\left(\mathcal{U}\left(\mathcal{P}_{4}\right) \cap \mathcal{E}^{C}\right)_{v}$. Moreover, the permutation $\beta_{4}$ is invertible, with inverse $\beta_{4}^{-1}$ defined by

$$
E^{\beta_{4}^{-1}}=\left(\mathcal{R}_{i_{2}} \cap E^{\theta^{-2}}\right) \cup\left(E \backslash \mathcal{R}_{i_{2}}\right),
$$

for all $E \in\left(\mathcal{U}\left(\mathcal{P}_{4}\right) \cap \mathcal{E}^{C}\right)_{v}$, where $i_{1}=i_{1}(\mathcal{O})$ and $i_{2}=i_{2}(\mathcal{O})$ for the orbit $\mathcal{O}$ of $\theta$ on $\mathcal{V}^{(k)}$ containing $E$. We conclude that $\left|\left(\mathcal{U}\left(\mathcal{P}_{4}\right) \cap \mathcal{E}\right)_{v}\right|=\left|\left(\mathcal{U}\left(\mathcal{P}_{4}\right) \cap \mathcal{E}^{C}\right)_{v}\right|$, and hence

$$
\operatorname{setval}_{\mathcal{U}\left(\mathcal{P}_{4}\right) \cap \mathcal{E}}(v)=\operatorname{setval}_{\mathcal{U}\left(\mathcal{P}_{4}\right) \cap \mathcal{E}^{C}}(v) .
$$

Observe that

$$
\operatorname{val}_{\mathcal{Y}_{k}}(v)=\sum_{i=1}^{4} \operatorname{setval}_{\mathcal{U}\left(\mathcal{P}_{i}\right) \cap \mathcal{E}}(v)=\sum_{i=1}^{4} \operatorname{setval}_{\mathcal{U}\left(\mathcal{P}_{i}\right) \cap \mathcal{E}^{C}}(v)=\operatorname{val}_{\mathcal{Y}_{k}^{C}}(v) .
$$

Since $j^{\prime}$ was an arbitrary element of $\mathbb{Z}_{m}$, we conclude that $\operatorname{val}_{\mathcal{Y}_{k}}(v)=\operatorname{val}_{\mathcal{Y}_{k}^{C}}(v)$ for all $v \in \mathcal{R}=\cup_{j \in \mathbb{Z}_{m}} \mathcal{R}_{j}$, and hence for all $v \in \mathcal{V}=\mathcal{R} \cup\{\infty\}$. Thus Lemma 3.1(1) implies that $\mathcal{Y}_{k}$ is 1 -subset-regular.

It should be noted that Lemma 3.3 was proved previously for the case where $a=2$. Rao handled the case where $a=2$ and $k=2$ in [13], and Potočnik and S̆ajna handled the case where $a=2$ and $k=3$ in [11].

We are ready to prove the sufficiency of condition (4) in Theorem 1.1. Lemma 3.4 demonstrates the existence of a 1-subset-regular self-complementary uniform hypergraph of rank $k$ and order $n$ for every pair $(n, k)$ satisfying condition (1).

Lemma 3.4. Let $a, k, m$, and $s$ be positive integers such that $a \geq 2$ and $s<k_{\left[2^{a}\right]}$. Let $\mathcal{R}=\mathbb{Z}_{m 2^{a}}$, let $S=\left\{\infty_{1}, \infty_{2}, \ldots, \infty_{s}\right\}$ such that $S \cap \mathcal{R}=\emptyset$, and let $\mathcal{V}=S \cup \mathcal{R}$. There exists a 1-subset-regular self-complementary $k$-hypergraph on $\mathcal{V}$ with antimorphism

$$
\theta=\left(\infty_{1}\right)\left(\infty_{2}\right) \cdots\left(\infty_{s}\right) \prod_{j=0}^{m-1}\left(j 2^{a}, j 2^{a}+1, \ldots,(j+1) 2^{a}-1\right) .
$$

Proof: Fix positive integers $a$ and $m$ such that $a \geq 2$. We prove that there exists a 1 -subset-regular self-complementary $k$-hypergraph on $\mathcal{V}$ with antimorphism $\theta$ for all positive integers $k$ and $s$ such that $1 \leq s<k_{\left[2^{a}\right]}$. The proof is 
by induction on $s$.

Base Step: $s=1$. In this case, since $s<k_{\left[2^{a}\right]}$, we have $k_{\left[2^{a}\right]} \geq 2$, and so the existence of a 1-subset-regular self-complementary $k$-hypergraph on $\mathcal{V}=$ $\left\{\infty_{1}\right\} \cup \mathbb{Z}_{m 2^{a}}$ with antimorphism

$$
\theta=\left(\infty_{1}\right) \prod_{j=0}^{m-1}\left(j 2^{a}, j 2^{a}+1, \ldots,(j+1) 2^{a}-1\right)
$$

follows from Lemma 3.3. Hence the result holds for $s=1$.

Induction Step: Suppose $s>1$, and assume that there exists a 1-subset-regular self-complementary $\hat{k}$-hypergraph $Z_{\hat{k}}$ on

$$
\hat{\mathcal{V}}=\left\{\infty_{1}, \ldots, \infty_{s-1}\right\} \cup \mathbb{Z}_{m 2^{a}}
$$

with antimorphism

$$
\hat{\theta}=\left(\infty_{1}\right) \cdots\left(\infty_{s-1}\right) \prod_{j=0}^{m-1}\left(j 2^{a}, j 2^{a}+1, \ldots,(j+1) 2^{a}-1\right),
$$

for all $\hat{k}$ such that $1 \leq s-1<\hat{k}_{\left[2^{a}\right]}$.

Let $k$ be a positive integer such that $s<k_{\left[2^{a}\right]}$. We will construct a 1 -subsetregular self-complementary $k$-hypergraph on $\mathcal{V}$ with antimorphism $\theta$. Now $1 \leq$ $s-1<k_{\left[2^{a}\right]}$ and so by the induction hypothesis, there exists a 1-subset-regular self-complementary $k$-hypergraph $Z_{k}$ on $\hat{\mathcal{V}}$ with antimorphism $\hat{\theta}$. Moreover, since $s \geq 2$, we have $k_{\left[2^{a}\right]} \geq 3$, and so $(k-1)_{\left[2^{a}\right]}=k_{\left[2^{a}\right]}-1$. This implies that $1 \leq s-1<(k-1)_{\left[2^{a}\right]}$, and so by the induction hypothesis, there also exists a 1-subset-regular self-complementary $(k-1)$-hypergraph $Z_{k-1}$ on $\hat{\mathcal{V}}$ with antimorphism $\hat{\theta}$.

Let $\mathcal{Z}_{k}$ be the $k$-hypergraph with vertex set $\mathcal{V}=\hat{\mathcal{V}} \cup\left\{\infty_{s}\right\}$ and edge set

$$
\mathcal{E}=\mathcal{E}\left(Z_{k}\right) \bigcup\left\{\left\{\infty_{s}\right\} \cup E: E \in \mathcal{E}\left(Z_{k-1}\right)\right\} .
$$

Since $\left.\theta\right|_{\hat{\mathcal{V}}}=\hat{\theta} \in \operatorname{Ant}\left(Z_{k}\right) \cap \operatorname{Ant}\left(Z_{k-1}\right)$, and $\theta$ fixes $\infty_{s}$, it follows that $E \in \mathcal{E}$ if and only if $E^{\theta} \in \mathcal{E}^{C}$. Hence $\theta \in \operatorname{Ant}\left(\mathcal{Z}_{k}\right)$ and $\mathcal{Z}_{k}$ is self-complementary. Moreover, for all $v \in \hat{\mathcal{V}}$, we have

$$
\begin{aligned}
\operatorname{val}_{\mathcal{Z}_{k}}(v) & =\operatorname{val}_{Z_{k}}(v)+\operatorname{val}_{Z_{k-1}}(v) \\
& =\operatorname{val}_{Z_{k}^{C}}(v)+\operatorname{val}_{Z_{k-1}^{C}}(v) \\
& =\operatorname{val}_{\mathcal{Z}_{k}^{C}}(v) .
\end{aligned}
$$

Since the antimorphism $\theta$ fixes $\infty_{s}$, we also have $\operatorname{val}_{\mathcal{Z}_{k}}\left(\infty_{s}\right)=\operatorname{val}_{\mathcal{Z}_{k}^{C}}\left(\infty_{s}\right)$, and so $\operatorname{val}_{\mathcal{Z}_{k}}(v)=\operatorname{val}_{\mathcal{Z}_{k}^{C}}(v)$ for all $v \in \hat{\mathcal{V}} \cup\{\infty\}=\mathcal{V}$. Thus Lemma 3.1(1) implies that $\mathcal{Z}_{k}$ is 1 -subset-regular. 
Hence by induction on $s$, there exists a 1-subset-regular self-complementary $k$-hypergraph on $\mathcal{V}$ with antimorphism $\theta$ for every positive integer $s<k_{\left[2^{a}\right]}$.

Proof of Theorem 1.2: The necessity of condition (1) follows from Theorem 1.1. Suppose that $n$ satisfies condition (1). Then $n=m 2^{a}+s$ for some positive integers $a, m$, and $s$ such that $\max \left\{i: 2^{i} \mid k\right\}<a \leq \min \left\{i: 2^{i}>k\right\}$ and $1 \leq s<k_{\left[2^{a}\right]}$. If $a=1$, then $1 \leq s<k_{\left[2^{a}\right]}$ cannot hold, and so in this case the sufficiency of condition (1) holds vacuously. On the other hand, if $a \geq 2$, then the existence of a 1-subset-regular self-complementary $k$-hypergraph of order $n$ follows from Lemma 3.4, and so condition (1) is sufficient in this case also.

Theorem 2.1 shows that condition (1) is equivalent to condition (3), and so condition (3) is an alternative statement of the necessary and sufficient conditions of Theorem 1.2 on the order $n$ of a 1-subset-regular self-complementary $k$-uniform hypergraph, in terms of the binary representation of $k$.

\section{A Appendix}

The next two technical lemmas are used in the proof of Lemma 3.2 in Section 3.

Lemma A.1. Let $\alpha, i$, and $j$ be integers such that $0 \leq i, j \leq 2^{\alpha}, i+j$ is odd, and $\frac{1}{2^{\alpha}}\left(\begin{array}{c}2^{\alpha} \\ i\end{array}\right)\left(\begin{array}{c}2^{\alpha} \\ j\end{array}\right)$ is an integer. Then $\frac{1}{2^{\alpha}}\left(\begin{array}{c}2^{\alpha} \\ i\end{array}\right)\left(\begin{array}{c}2^{\alpha} \\ j\end{array}\right)$ is odd if and only if $i \in\left\{0,2^{\alpha}\right\}$ or $j \in\left\{0,2^{\alpha}\right\}$.

Proof: Since $i+j$ is odd, either $i$ or $j$ must be odd. First suppose that $i$ is odd. Now

$$
\frac{1}{2^{\alpha}}\left(\begin{array}{c}
2^{\alpha} \\
i
\end{array}\right)\left(\begin{array}{c}
2^{\alpha} \\
j
\end{array}\right)=\frac{1}{2^{\alpha}-i}\left(\begin{array}{c}
2^{\alpha}-1 \\
i
\end{array}\right)\left(\begin{array}{c}
2^{\alpha} \\
j
\end{array}\right)
$$

Since $i \leq 2^{\alpha}$ and $i$ is odd, any integer $r$ in the support of the binary representation of $i$ satisfies $r \leq \alpha-1$. Since $\left(2^{\alpha}-1\right)_{\left[2^{r+1}\right]} \geq i_{\left[2^{r+1}\right]}$ for all such $r$, it follows that $\left(\begin{array}{c}2^{\alpha}-1 \\ i\end{array}\right)$ is odd. Now if $j \notin\left\{0,2^{\alpha}\right\}$, then $\left(2^{\alpha}\right)_{\left[2^{r+1}\right]}=0<j_{\left[2^{r+1}\right]}$ for some $r$ in the support of the binary representation of $j$, which implies that $\left(\begin{array}{c}2^{\alpha} \\ j\end{array}\right)$ is even. Since $2^{\alpha}-i$ is odd, this implies that the integer $\frac{1}{2^{\alpha}}\left(\begin{array}{c}2^{\alpha} \\ i\end{array}\right)\left(\begin{array}{c}2^{\alpha} \\ j\end{array}\right)=$ $\frac{1}{2^{\alpha}-i}\left(\begin{array}{c}2^{\alpha}-1 \\ i\end{array}\right)\left(\begin{array}{c}2^{\alpha} \\ j\end{array}\right)$ is even. On the other hand, if $j \in\left\{0,2^{\alpha}\right\}$, then $\left(\begin{array}{c}2^{\alpha} \\ j\end{array}\right)=1$, and so $\frac{1}{2^{\alpha}}\left(\begin{array}{c}2^{\alpha} \\ i\end{array}\right)\left(\begin{array}{c}2^{\alpha} \\ j\end{array}\right)=\frac{1}{2^{\alpha}-i}\left(\begin{array}{c}2^{\alpha}-1 \\ i\end{array}\right)\left(\begin{array}{c}2^{\alpha} \\ j\end{array}\right)=\frac{1}{2^{\alpha}-i}\left(\begin{array}{c}2^{\alpha}-1 \\ i\end{array}\right)$ is an odd integer. Thus if $i$ is odd, then $\frac{1}{2^{\alpha}}\left(\begin{array}{c}2^{\alpha} \\ i\end{array}\right)\left(\begin{array}{c}2^{\alpha} \\ j\end{array}\right)$ is odd if and only if $j \in\left\{0,2^{\alpha}\right\}$.

By a symmetric argument, if $j$ is odd, then $\frac{1}{2^{\alpha}}\left(2^{\alpha}{ }_{i}\right)\left(\begin{array}{c}2^{\alpha} \\ j\end{array}\right)$ is odd if and only if $i \in\left\{0,2^{\alpha}\right\}$.

Lemma A.2. Let $r$ be a nonnegative integer. Suppose that $\lambda_{1}, \lambda_{2}, \ldots, \lambda_{n}$ is a sequence of integers such that $0 \leq \lambda_{i} \leq r$ for all $i \in\{1,2, \ldots, n\}$. Then there is a function $v:\left\{\lambda_{1}, \lambda_{2}, \ldots, \lambda_{n}\right\} \rightarrow\{-1,1\}$ such that $0 \leq \sum_{i=1}^{n} \lambda_{i} v\left(\lambda_{i}\right) \leq r$. 
Proof: The proof is by induction on $n$. If $n=1$, then take $v\left(\lambda_{1}\right)=1$. If $n=2$, then if $\lambda_{1} \leq \lambda_{2}$, take $v\left(\lambda_{1}\right)=-1$ and $v\left(\lambda_{2}\right)=1$, and if $\lambda_{1} \geq \lambda_{2}$, take $v\left(\lambda_{1}\right)=1$ and $v\left(\lambda_{2}\right)=-1$. Hence the result holds when $n \in\{1,2\}$.

Let $n>2$ and suppose the result holds for all such sequences of length $n-1$. Let $\lambda_{1}, \lambda_{2}, \ldots, \lambda_{n}$ be a sequence of integers such that $0 \leq \lambda_{i} \leq r$ for all $i \in\{1,2, \ldots, n\}$. By the induction hypothesis, there is a function $v^{\prime}$ : $\left\{\lambda_{1}, \ldots, \lambda_{n-1}\right\} \rightarrow\{-1,1\}$ such that $\sum_{i=1}^{n-1} \lambda_{i} v^{\prime}\left(\lambda_{i}\right)=\lambda$ for some $\lambda$ such that $0 \leq \lambda \leq r$. By the base case $n=2$, there is a function $\hat{v}:\left\{\lambda, \lambda_{n}\right\} \rightarrow\{-1,1\}$ such that $0 \leq \lambda \hat{v}(\lambda)+\lambda_{n} \hat{v}\left(\lambda_{n}\right) \leq r$. Let $v$ be the function $v:\left\{\lambda_{1}, \ldots, \lambda_{n}\right\} \rightarrow\{-1,1\}$ such that $v\left(\lambda_{i}\right)=\hat{v}(\lambda) v^{\prime}\left(\lambda_{i}\right)$ for $i \in\{1,2, \ldots, n-1\}$, and $v\left(\lambda_{n}\right)=\hat{v}\left(\lambda_{n}\right)$. Then $\sum_{i=1}^{n} \lambda_{i} v\left(\lambda_{i}\right)=\lambda \hat{v}(\lambda)+\lambda_{n} \hat{v}\left(\lambda_{n}\right)$ and so $0 \leq \sum_{i=1}^{n} \lambda_{i} v\left(\lambda_{i}\right) \leq r$ as required. The result follows by induction.

\section{References}

[1] S. Ajoodani-Namini, All block designs with $b=\left(\begin{array}{l}v \\ k\end{array}\right) / 2$ exist, Discrete Math. 179 (1998), 27-35.

[2] S. Ajoodani-Namini and G.B. Khosrovshahi, More on halving the complete design, Discrete Math. 135 (1994), 29-37.

[3] S. Ajoodani-Namini and G.B. Khosrovshahi, Combining t-designs, J. Combin. Theory, Ser. A 58 (1991), 26-34.

[4] S. Ajoodani-Namini and G.B. Khosrovshahi, A new basis for trades, SIAM J. Discrete Math. 3 (1990), 364-372.

[5] W.O. Alltop, Extending t-designs, J. Combin. Theory, Ser. A 18 (1975), $177-186$.

[6] Z. Baranyai, On the factorizations of complete uniform hypergraphs, Finite and Infinite Sets, Colloq. Math. Soc. Janos Bolyai 10 (1975), 91-108, North Holland, Amsterdam.

[7] M. Dehon, On the existence of 2-designs $S_{\lambda}(2,3, v)$ without repeated blocks, Discrete Math. 43 (1983), 155-171.

[8] A. Hartman, Halving the complete design, Ann. Discrete Math. 34 (1987), 207-224.

[9] G.B. Khosrovshahi and B. Tayfeh-Rezaie, Root cases of large sets of $t$ designs, Discrete Math. 263 (2003), 143-155.

[10] M. Knor and P. Potočnik, A note on 2-subset-regular self-complementary 3-uniform hypergraphs (2008). To appear in Ars Combin.

[11] P. Potočnik and M. Šajna, The existence of regular selfcomplementary 3-uniform hypergraphs, Discrete Math. (2008), doi:10.1016/j.disc.2008.01.026. 
[12] P. Potočnik and M. Šajna, Vertex-transitive self-complementary uniform hypergraphs. European J. Combin. 30 (2009), 327-337.

[13] S.B. Rao, On regular and strongly-regular self-complementary graphs, Discrete Math. 54 (1985), 73-82.

[14] L. Teirlink, Locally trivial $t$-designs and $t$-designs without repeated blocks, Discrete Math. 77 (1989), 345-356. R.

[15] M. Wilson, Decomposition of complete graphs into subgraphs isomorphic to a given graph, Congressus Numerantium XV (1975), 647-659. 